\title{
Improved functional outcome in NTOS patients following resection of the subclavius muscle with radiological signs of nerve impingement: indication of participation of the subclavius in brachial plexus compression
}

\author{
Yanxi Liu, MD, Zhan Zhang, MD, Jiangbo Wang, MD, Guangzhi Wu, MD, Wei Yu, MD, and \\ Shusen Cui, MD \\ Department of Hand Surgery, China-Japan Union Hospital of Jilin University, Changchun, Jilin, People's Republic of China
}

OBJECTIVE Both clinical and radiological reports have suggested that the subclavius, a muscle in the costoclavicular space of the thoracic outlet, participates in neurogenic thoracic outlet syndrome (NTOS) in some instances, especially during movements narrowing the costoclavicular space. Magnetic resonance imaging can identify subclavius muscles with signs of nerve impingement, yet the impact of the subclavius in such situations remains unclear. Therefore, the authors investigated whether dividing or sparing the subclavius characterized by nerve impingement on MRI would affect surgical outcomes.

METHODS In this retrospective nonrandomized study, authors analyzed all NTOS patients with a subclavius muscle characterized by nerve impingement on MRI (loss of normal fat planes surrounding the brachial plexus) in the period between March 2010 and November 2016. Patients were divided into two groups: the sparing group, in which patients had undergone conventional supraclavicular scalenectomy and first rib resection (FRR), and the dividing group, in which patients had undergone scalenectomy, FRR, and subclavius dividing using a modified supraclavicular incision. The Disabilities of the Arm, Shoulder and Hand (DASH) questionnaire, a shoulder range of motion subscale (DASH items $6,12-15$, and 19) concerning overhead activities that can significantly narrow the costoclavicular space, postoperative MRI studies, and patient self-assessments were used to assess surgical outcomes. Univariate and multivariate analyses were conducted to identify independent factors associated with subscale scores.

RESULTS From a total of 261 patients screened, 71 were eligible for study inclusion. Compared with the sparing group (33 patients), the dividing group (38 patients) had similar postoperative DASH scores and self-assessments but better subscale scores $(9.50 \pm 2.76$ vs $11.94 \pm 2.87, p=0.0005)$. Postoperative MRI on hyperabduction showed that the brachial plexus became surrounded by normal fat tissue in the costoclavicular space in the diving group but still had signs of impingement from the untreated subclavius muscle in the sparing group. This observation agreed with a better functional recovery in terms of overhead activities in the dividing group, which was reflected by better subscale scores. Multivariate analyses indicated that the type of treatment and symptom duration prior to surgery influenced the subscale scores independently.

CONCLUSIONS This study revealed that an untreated radiological nerve-compressing subclavius muscle could lead to a relatively lower degree of recovery in the ability to perform overhead activities for NTOS patients postoperatively, suggesting that such subclavius muscles may participate in positional brachial plexus compression during movements narrowing the costoclavicular space. Dividing the muscles could decompress the costoclavicular space more effectively and may lead to better functional recovery.

https://thejns.org/doi/abs/10.3171/2018.5.JNS18429

KEYWORDS neurogenic thoracic outlet syndrome; subclavius muscle; magnetic resonance imaging; radiological signs of nerve impingement; functional outcome; peripheral nerve

ABBREVIATIONS DASH = Disabilities of the Arm, Shoulder and Hand; FRR = first rib resection; NTOS = neurogenic thoracic outlet syndrome; PT = physical therapy. SUBMITTED February 12, 2018. ACCEPTED May 10, 2018.

INCLUDE WHEN CITING Published online November 9, 2018; DOI: 10.3171/2018.5.JNS18429. 
$\mathrm{N}$ EUROGENIC thoracic outlet syndrome (NTOS) occurs when there is insufficient space for the brachial plexus. ${ }^{3}$ Neurological symptoms in the arms can be triggered or worsened by movement that narrows the thoracic outlet, such as reaching overhead and lifting heavy objects. ${ }^{17}$ Factors including cervical ribs, anatomical variations in first ribs, scalene and pectoralis minor muscle anomalies, and various fibrous bands contribute to the multifaceted pathology of NTOS. ${ }^{20}$

Recently, it was reported that the subclavius, a muscle originating from the first rib and inserting at the clavicle, was thicker and compromised passage of the brachial plexus in the costoclavicular space of the thoracic outlet in NTOS patients compared with that in healthy subjects. ${ }^{9}$ Subclavius muscles can dynamically compress the brachial plexus passing beneath them during movements narrowing the costoclavicular space such as arm hyperabduction, especially when variations such as anomalous coursing or hypertrophy are present in the muscles. ${ }^{6,7,14,16,24,26}$

Investigators in several studies have adopted modified treatments involving the subclavius $2,18,25,33,34$ for NTOS, and those in two studies achieved improved treatment outcomes after adding subclavius blocks to nonsurgical treatment ${ }^{18}$ or resecting the subclavius they found to be hypertrophic. ${ }^{2}$ However, because of concurrent changes in other techniques in these studies, ${ }^{2,18}$ it was unclear whether the improved outcome resulted directly from treating the subclavius.

Magnetic resonance imaging can identify subclavius muscles characterized by nerve impingement (loss of fat planes surrounding the nerves). ${ }^{9,10,36}$ However, it is uncertain whether such a subclavius muscle genuinely compresses the nerves. Symptoms of NTOS can be caused by multiple structures concurrently, and the contribution of the subclavius cannot be confirmed by specific upper extremity complaints or physical findings. For instance, though physical examination via the costoclavicular maneuver can suggest nerve entrapment in the costoclavicular space, ${ }^{28}$ it cannot indicate whether the compression comes from the first rib, the subclavius, or both.

Involvement of the subclavius muscle in the development of NTOS, which is still unclear, especially matters to surgeons given the difference in handling subclavius muscles between transaxillary and supraclavicular approaches. While the subclavius is unexposed in supraclavicular decompressions, it is routinely cut in transaxillary approaches to make room for first rib resection (FRR), ${ }^{31}$ unintentionally relieving tension caused by the subclavius.

Though preoperative imaging studies, such as MRI, can help surgeons identify subclavius muscles likely to be involved in NTOS, the participation of a "radiologically nerve-compressing" subclavius in NTOS needs to be better understood to provide stronger evidence to assist surgical decision-making. Therefore, the participation of subclavius muscle in brachial plexus compression, which is difficult to determine through a patient's symptoms or diagnostic tests, was evaluated in the present study by analyzing whether dividing or sparing the muscle affects surgical outcomes, particularly in terms of the ability to perform overhead activities, which can significantly narrow the costoclavicular space.

\section{Methods}

This study was approved by the Institutional Review Board of China-Japan Union Hospital of Jilin University. Written informed consent was obtained from the patients included.

\section{Study Design and Patient Selection}

Retrospective review was performed on 261 NTOS patients treated in the period between March 1, 2010, and November 31, 2016. None of the patients had undergone previous decompression surgery, and all were considered as surgical candidates because of failed conservative treatment. Eligible for inclusion in the study were those patients with a subclavius muscle showing radiological evidence of nerve impingement and without coexisting conditions (vascular thoracic outlet syndrome, cervical disc disease, distal nerve entrapment, frozen shoulder, or neurogenic pectoralis minor syndrome) that might confound NTOS symptoms. Patients who missed follow-up assessment or did not follow the postoperative physical therapy (PT) protocol were excluded from analysis. Detailed descriptions of our NTOS diagnostic criteria are provided in our Supplemental Material.

Patients were divided into two groups: the sparing group, in which patients had undergone a conventional supraclavicular approach, and the dividing group, in which patients had undergone a modified supraclavicular approach (Fig. 1). Although transaxillary approaches enable subclavius muscle division (subclavius muscle attachments have to be separated from the first rib to expose the anterior part of the rib, which is subsequently removed), it was not adopted for the dividing group because it has other major differences from supraclavicular approaches that can confound results, for example, the extent of scalenectomy (partial vs total) and the segment of rib removal (anterior vs posterior). ${ }^{18}$ Patients were informed of the potential advantages and disadvantages of both procedures as well as possible complications. Briefly, the conventional supraclavicular approach is a well-developed approach with proven effective results in most patients without resection of the subclavius muscles. The modified approach enables subclavius division but requires a longer incision that extends to the deltopectoral groove. After the supraclavicular approach, common complications such as pneumothorax, long thoracic, and phrenic nerve injuries can occur. The modified approach can cause additional complications such as injuries to the cephalic vein and branches of the lateral pectoral nerve. Patients decided which approach to undergo.

\section{MRI Protocol and Analysis}

Magnetic resonance imaging examination was performed using a 3-T unit (Magnetom Skyra, Siemens) and a 3-mm slice thickness, with the patient's arms alongside the body and overhead (abducted to about $130^{\circ}$ and external rotation). ${ }^{8,9}$ For analysis, we used T1-weighted sequences, which have better soft tissue depiction. ${ }^{8,9}$

Patients with effacement of fat tissue (disappearance of the high intensity signal that represents the fat) between the brachial plexus and the subclavius muscle demon- 
A

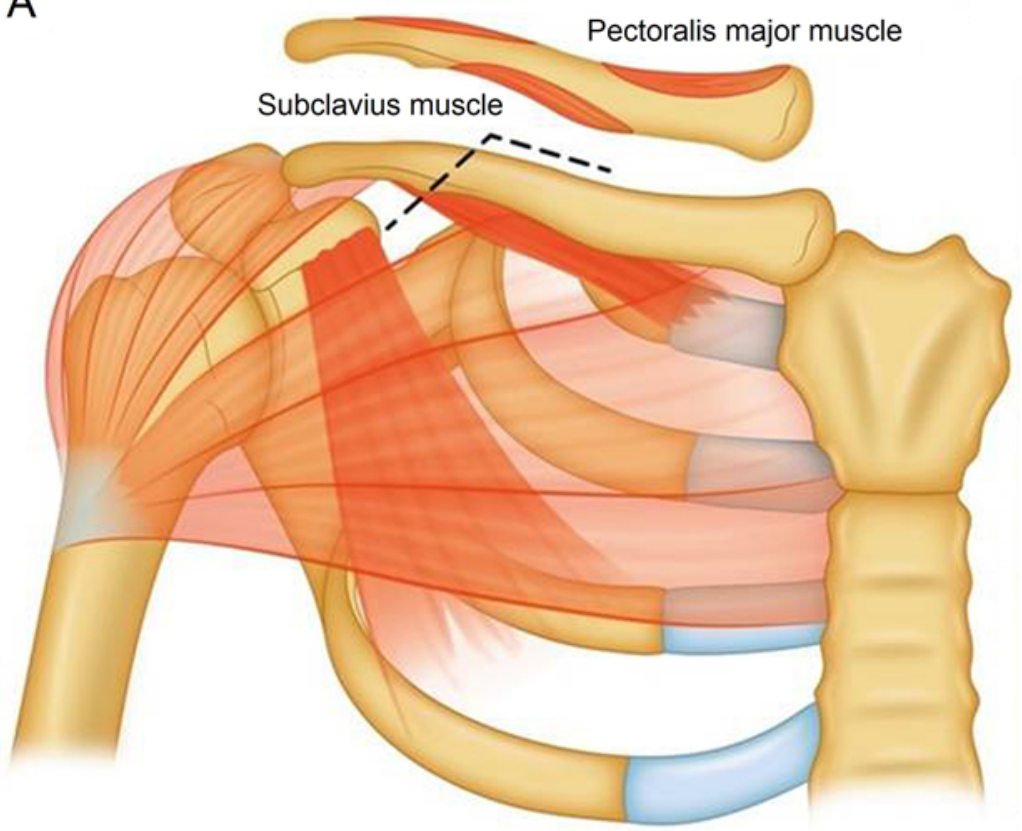

B

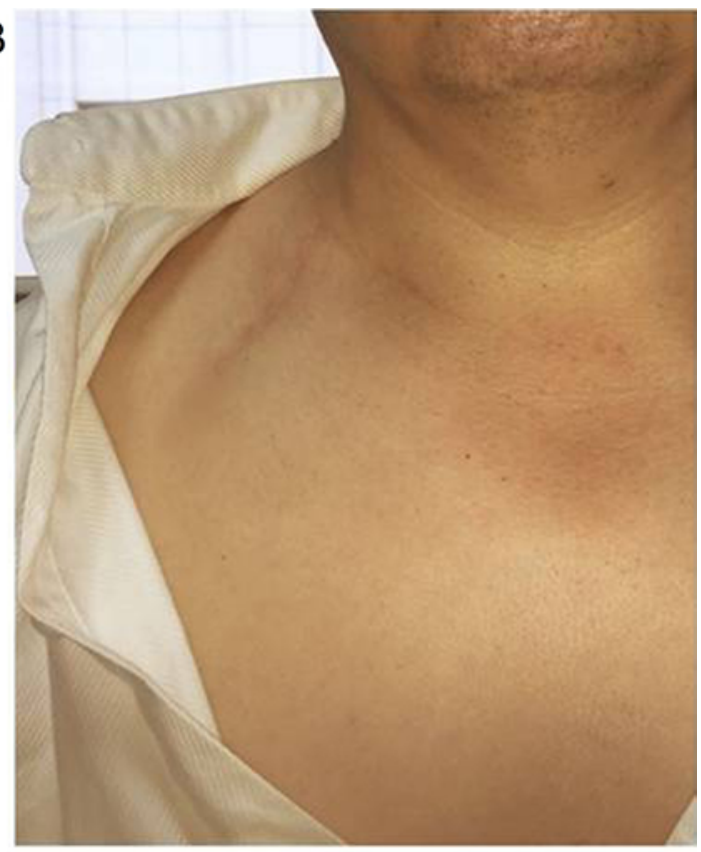

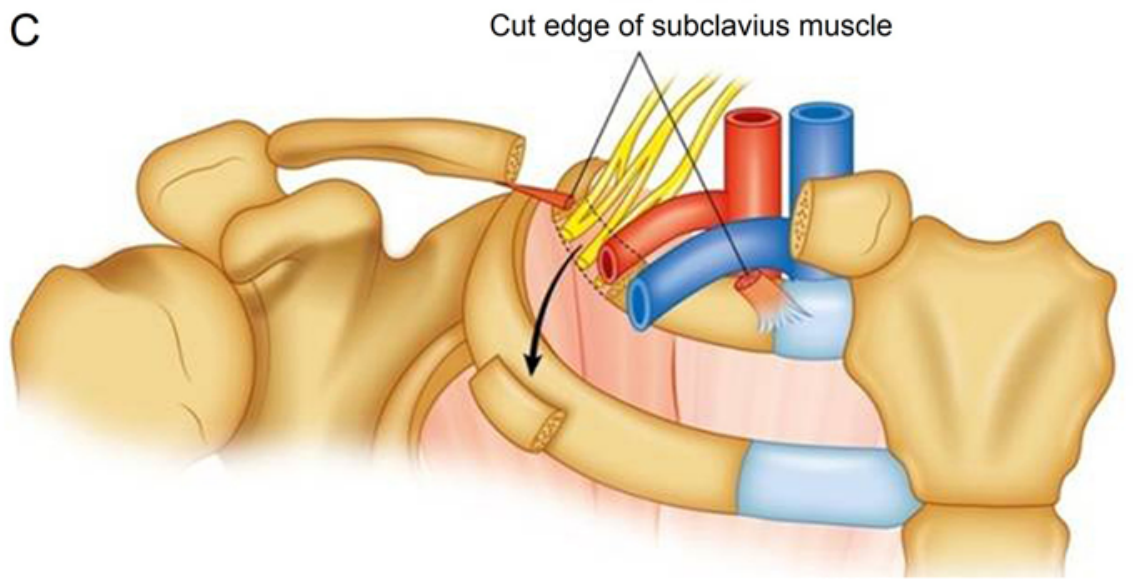

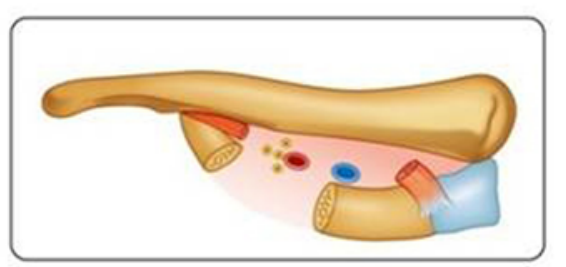

FIG. 1. The modified supraclavicular approach. A: Attachment points of the relevant depicted muscles onto the clavicle and the incision (dashed line) for the modified supraclavicular approach. The lateral end of the conventional supraclavicular incision was extended downward over the deltopectoral groove, which exposes both supra- and infraclavicular areas. Note that the subclavius muscle can be accessed through the deltopectoral groove. B: A patient surgically treated with the modified supraclavicular approach. C: Part of the clavicle is removed in this drawing for a clearer view. The modified incision adopted in the dividing group allows for division and partial excision of both the "floor" (first rib) and the "roof" (subclavius) of the costoclavicular space. Artwork by Elsevier Webshop. Copyright Shusen Cui. Published with permission. Figure is available in color online only.

strated on both sagittal and coronal views were considered to have subclavius muscles with signs of nerve impingement. Effacement of fat tissue on at least three consecutive coronal sequences (a length of $9 \mathrm{~mm}$ according to section thickness) was required to minimize potential falsepositives.

Cases were referred to as radiologically negative if MRI demonstrated the absence of contact between the brachial plexus and the subclavius muscle in any of the imaging planes in either hyperabduction or neutral positions (Fig. 2). Cases were referred to as radiologically positive if MRI demonstrated signs of nerve impingement on hyperabduction but not in the neutral position (Fig. 3) or at both positions (Fig. 4). Two radiologists independently judged whether there was radiological evidence of nerve impingement by the subclavius muscle, and if there was disagreement between the two, a third radiologist participated in making the final decision.

\section{Intervention}

The sparing group underwent the classic supraclavicular anterior and middle scalenectomy as well as FRR. The dividing group underwent scalenectomy, FRR, and subclavius muscle dividing using a modified supraclavicular incision depicted in the Atlas of Peripheral Nerve Surgery (Fig. 1).$^{19}$ In this modified approach, the lateral end of the incision was extended downward over the deltopectoral 

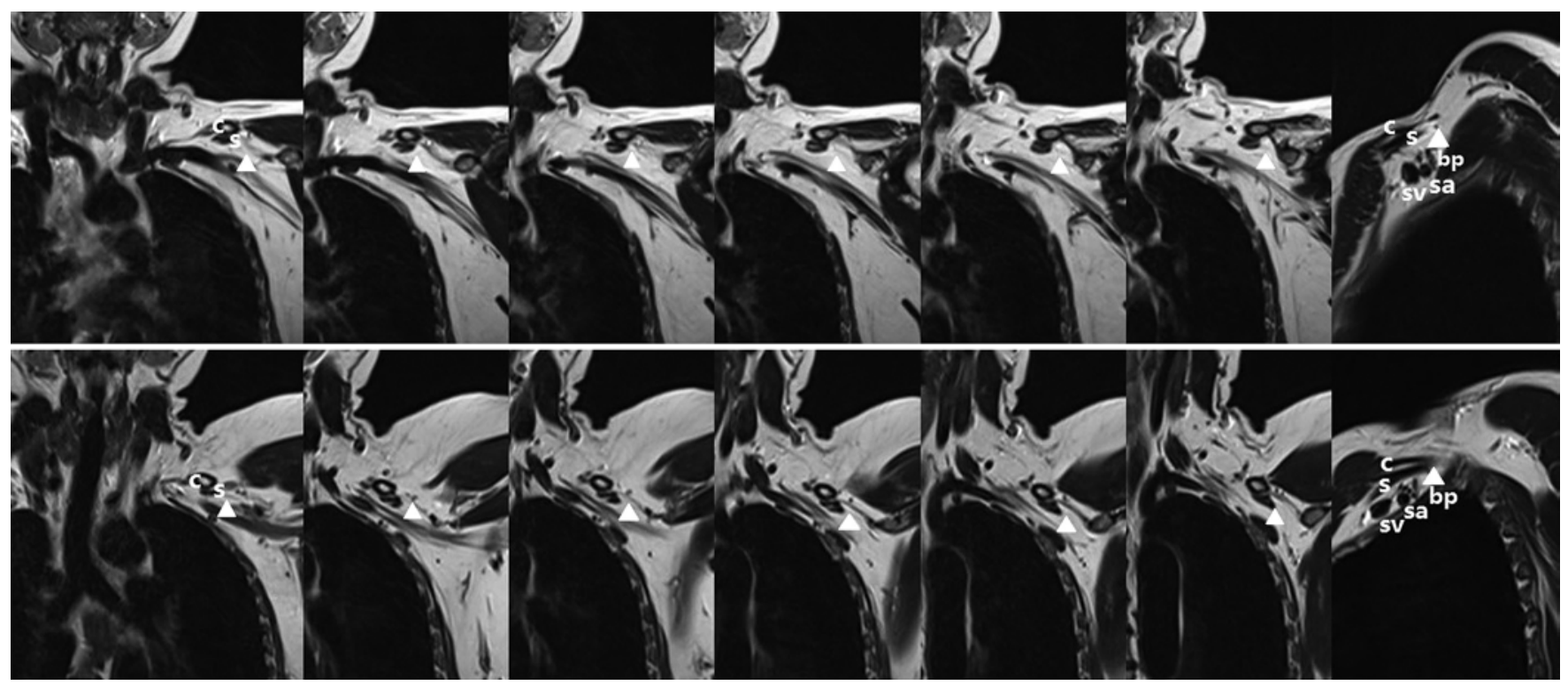

FIG. 2. Magnetic resonance imaging studies in NTOS patients with subclavius muscles that do not have radiological signs of nerve impingement; there is no contact between the brachial plexus and the subclavius muscle on any slices in either the neutral position (upper) or hyperabduction (lower). The brachial plexus is surrounded by fat tissues (high signal intensity) in the costoclavicular space. Triangles denote the normal existence of fat tissue surrounding the brachial plexus. $b p=$ brachial plexus; $c=c$ clavicle; $s=$ subclavius; sa = subclavian artery; sv = subclavian vein.

groove, which exposed both the supra- and infraclavicular regions and enabled subclavius muscle dividing. Detailed surgical techniques are shown in Supplemental Fig. 1.

The same postoperative PT protocol (Supplemental Material) was applied for both groups. Patients with poor compliance with PT, which was defined as completing less than $50 \%$ of the total required amount of PT (fewer than
3 days a week or less than one whole set per day) were excluded from analysis, as compliance with PT also affects functional outcomes. ${ }^{13,23}$

\section{Surgical Outcomes}

Postoperative functional outcome was assessed using the Disabilities of the Arm, Shoulder and Hand (DASH)

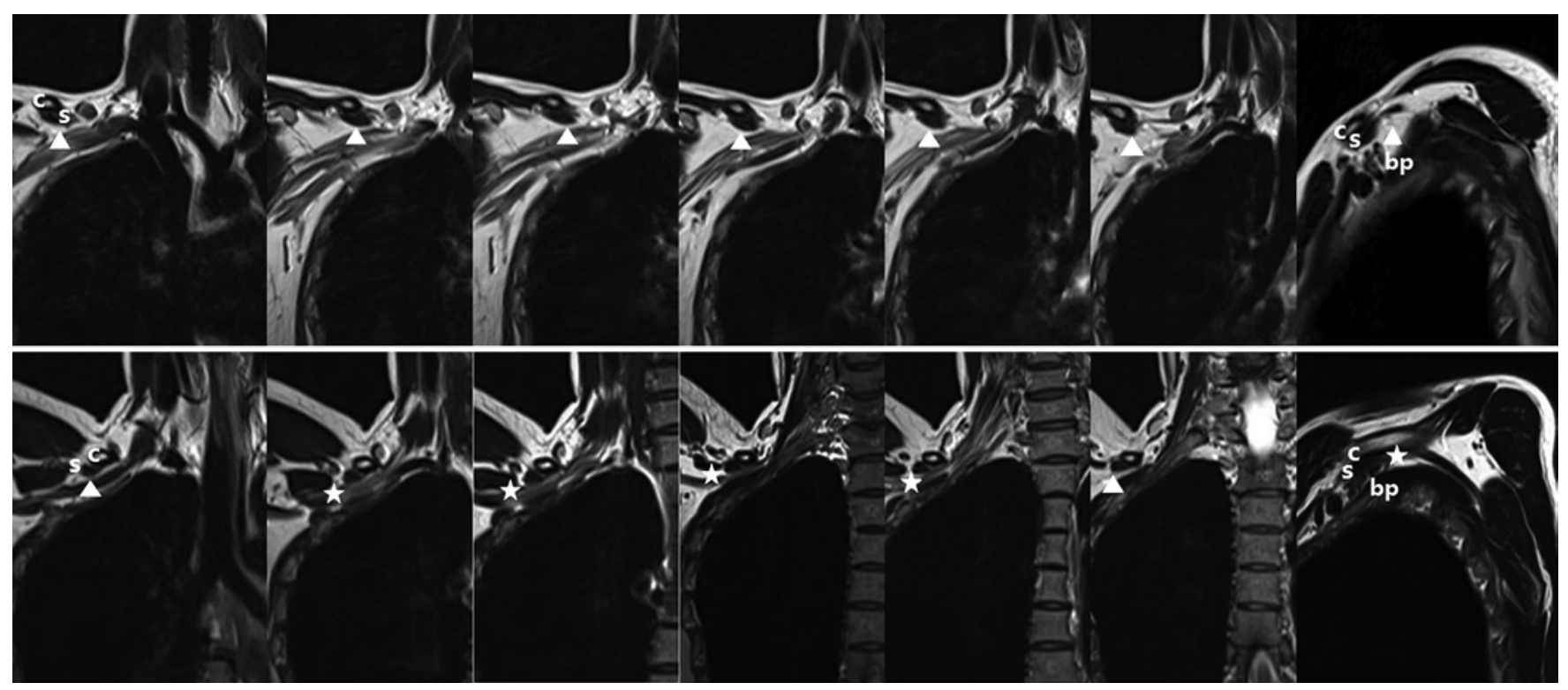

FIG. 3. Magnetic resonance imaging studies in NTOS patients with subclavius muscles showing radiological signs of nerve impingement on hyperabduction (lower) but not in the neutral position (upper). Stars denote the disappearance of the fat plane between the subclavius muscle and the brachial plexus. Triangles denote the normal existence of fat tissue surrounding the brachial plexus. $\mathrm{bp}=$ brachial plexus; $\mathrm{c}=$ clavicle; $\mathrm{s}=$ subclavius. 

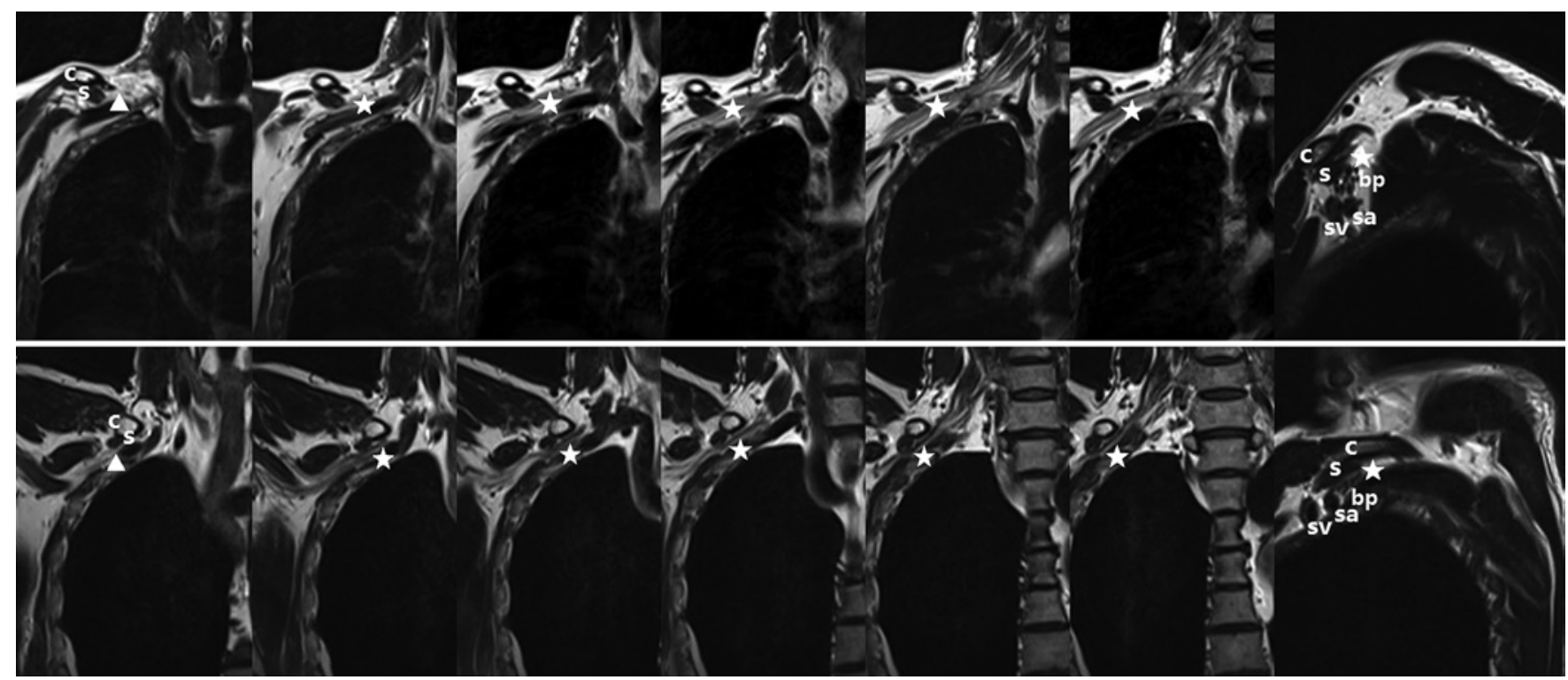

FIG. 4. Magnetic resonance imaging studies in NTOS patients with subclavius muscles showing radiological signs of nerve impingement in both the neutral position (upper) and hyperabduction (lower). Stars denote the disappearance of the fat plane between the subclavius muscle and the brachial plexus. Triangles denote the normal existence of fat tissue surrounding the brachial plexus. $\mathrm{bp}=$ brachial plexus; $\mathrm{c}=$ clavicle; $\mathrm{s}$ = subclavius; $\mathrm{sa}=$ subclavian artery; sv = subclavian vein.

questionnaire, each patient's self-assessment (reported as resolved, markedly improved, fair, or poor, depending on the degree of overall recovery; detailed criteria in Supplemental Material), and a shoulder range of motion subscale (DASH items 6, 12-15, and 19) established by factor analysis on the dimensionality of DASH..$^{12}$ All of the subscale items involve overhead activities: 6, placing an object on a shelf above your head; 12 , changing a light bulb overhead; 13, washing or blowing dry your hair; 14, washing your back; 15, putting on a pullover sweater; and 19, recreational activities in which you move your arm freely (e.g., badminton). Each item is scored from 1 to 5, with higher scores indicating greater disability. Assessments at the latest follow-up were used for analysis. Magnetic resonance imaging reexamination was performed 3 months postoperatively to evaluate radiological changes. For the sparing group, the relation between the left subclavius muscle and brachial plexus was assessed according to the same criteria used preoperatively. For the dividing group, whether the brachial plexus was free from compression in the costoclavicular space (existence of surrounding fat tissue on all the sagittal and coronal sequences) was assessed.

\section{Statistical Analysis}

Normality was assessed with the Shapiro-Wilk test. Normally distributed data were expressed as the means \pm standard deviation and were compared with an independent t-test. Nonnormally distributed data were presented as medians (range) and compared with a Mann-Whitney U-test. Categorical variables were compared with the chisquare test and Fisher's exact test. Univariate and multivariate analyses were conducted to identify factors independently associated with scale scores. The following parameters were examined: age, sex, trauma history be- fore symptom onset, duration of the presence of symptoms prior to surgery, bony anomalies, result of scalene muscle blocks, and type of treatment (subclavius muscle dividing vs sparing). Significant $(p<0.05)$ or nearly significant $(p$ $<0.10$ ) variables in the univariate analysis were entered into a multivariate analysis using stepwise multiple regression. A p $<0.05$ (two-tailed) was considered statistically significant.

\section{Results}

\section{Study Population and Preoperative MRI}

From a total of 261 patients screened, radiological evidence of nerve impingement by the subclavius muscle was seen in 102 cases with $91.1 \%$ of them (93 cases) showing signs of impingement on hyperabduction but not in the neutral position (Fig. 3). We observed that in the neutral position, there was usually comfortable space between the subclavius muscle and the brachial plexus. As the arm reached overhead, however, the distance between the clavicle and first rib decreased drastically and the brachial plexus became much closer to, or even encroached upon, the subclavius.

Among the 102 radiologically positive cases, 82 patients had no coexisting conditions and were included for further study. Forty-four of these 82 patients chose the modified supraclavicular approach, forming the dividing group, whereas the remaining 38 patients chose the conventional supraclavicular approach, forming the sparing group. Three patients from the dividing group and 2 from the sparing group were excluded from analysis because of missing follow-up assessments. Furthermore, another 3 patients from each group were excluded as they did not strictly follow the PT protocol. Ultimately, 38 patients 


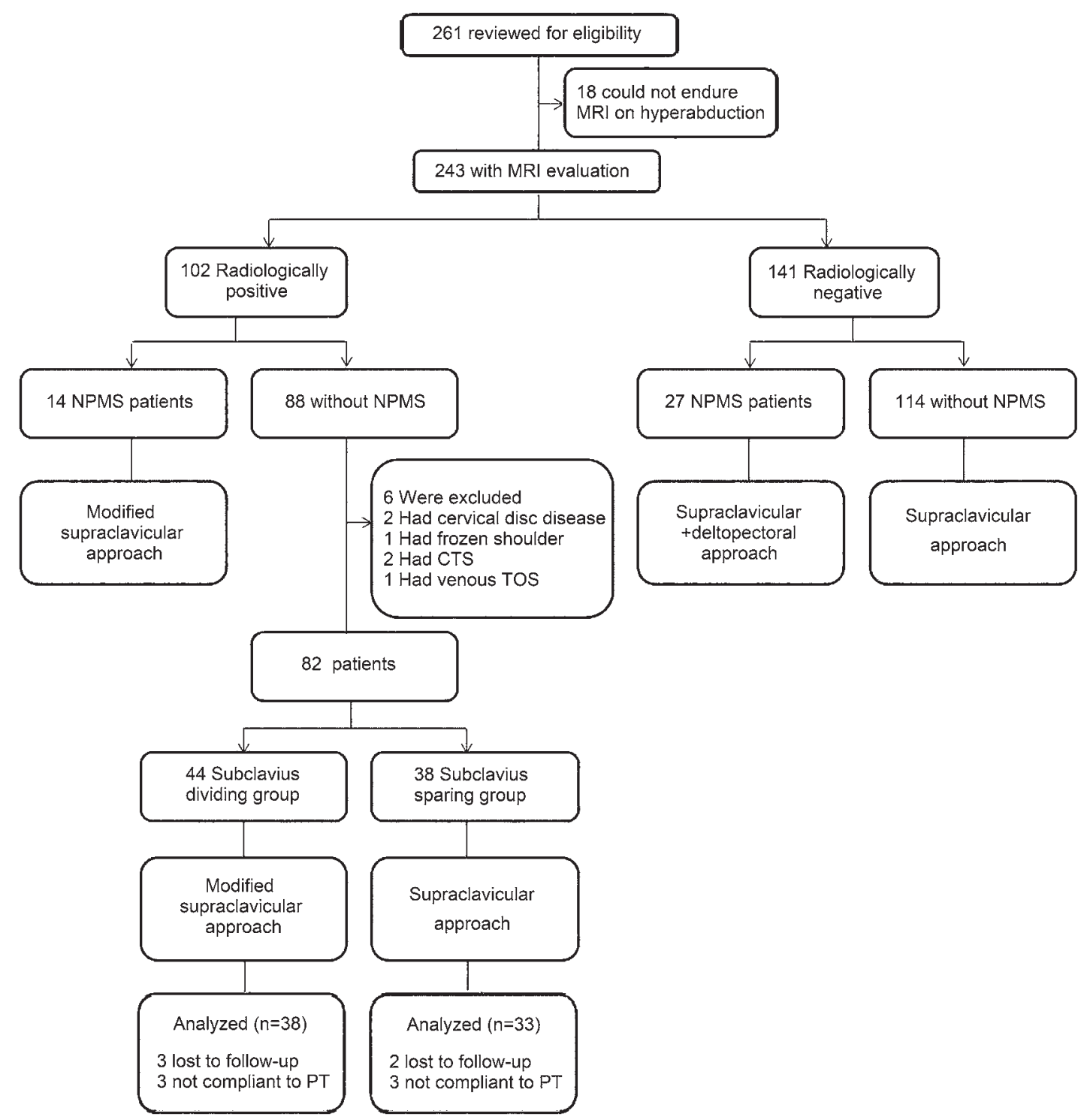

FIG. 5. Flow diagram of patients included in the study. CTS = carpal tunnel syndrome; NPMS = neurogenic pectoralis minor syndrome; TOS $=$ thoracic outlet syndrome.

from the dividing group and 33 from the sparing group were analyzed (Fig. 5).

These two treatment groups demonstrated similar demographic and clinical characteristics including patterns of neurogenic symptoms and results of physical examinations (Table 1). Mean preoperative DASH scores and subscale scores were similar between sparing and dividing groups $(51.39 \pm 10.74$ vs $53.38 \pm 9.63,18.52 \pm 2.28$ vs $18.90 \pm$ 3.38 , respectively). Three patients $(3 / 33$ [9.1\%]) in the sparing group and 6 in the dividing group (6/38 [15.8\%]) had radiological signs of nerve impingement by the subclavius muscle in both positions; the remaining patients had radiological compression only on hyperabduction.

\section{DASH Scores and Patient Self-Assessment Results}

Postoperative DASH scores and patient self-assessments were not significantly different between the two groups (Table 2). The DASH scores were significantly improved after surgery in both the sparing (29.94 \pm 9.96$)$ and dividing $(27.65 \pm 12.69)$ groups, and the mean extent of improvement in DASH scores (percentage change from baseline scores) was not significantly different between sparing $(41.74 \% \pm 5.82 \%)$ and dividing $(48.2 \% \pm 7.54 \%)$ groups $(\mathrm{p}=0.23)$. However, the dividing group had significantly lower subscale scores than the sparing group $(9.50 \pm$ 2.76 vs $11.94 \pm 2.87, p=0.0005$ ), reflecting a better ability to perform overhead activities.

\section{Postoperative MRI}

Magnetic resonance imaging reexamination was performed 3 months postoperatively to evaluate radiological changes (Fig. 6). For all 38 patients in the dividing group, there was no sign of nerve compression in the costoclavicular space in either the hyperabduction or neutral posi- 
TABLE 1. Baseline characteristics in subclavius sparing and dividing groups

\begin{tabular}{|c|c|c|c|}
\hline Parameter & $\begin{array}{c}\text { Subclavius } \\
\text { Sparing Group } \\
\text { (33) }\end{array}$ & $\begin{array}{l}\text { Subclavius } \\
\text { Dividing } \\
\text { Group (38) }\end{array}$ & $\begin{array}{c}p \\
\text { Value }\end{array}$ \\
\hline Mean age in yrs & $40.64 \pm 10.43$ & $42.26 \pm 10.74$ & 0.52 \\
\hline Sex & & & 0.65 \\
\hline Female & 20 & 21 & \\
\hline Male & 13 & 17 & \\
\hline Neck trauma before onset & & & 0.62 \\
\hline Yes & 12 & 16 & \\
\hline No & 21 & 22 & \\
\hline Bony anomaly & & & 0.90 \\
\hline Yes & 10 & 11 & \\
\hline No & 23 & 27 & \\
\hline $\begin{array}{l}\text { Median duration prior to op } \\
\text { in mos (range) }\end{array}$ & $30(3-126)$ & $17(5-250)$ & 0.42 \\
\hline \multicolumn{4}{|l|}{ Symptom } \\
\hline Weakness & 20 & 24 & \\
\hline Pain & 22 & 26 & \\
\hline Neck & 6 & 4 & \\
\hline Arm & 21 & 24 & \\
\hline Shoulder & 10 & 12 & \\
\hline Paresthesia & 33 & 38 & \\
\hline All 5 nerve roots & 19 & 10 & \\
\hline Lower plexus & 18 & 21 & \\
\hline Upper plexus & 6 & 7 & \\
\hline Muscle atrophy & 4 & 5 & \\
\hline \multicolumn{4}{|l|}{ Examination } \\
\hline Scalene tenderness & 25 & 23 & \\
\hline Positive EAST & 30 & 32 & \\
\hline Positive ULTT & 24 & 27 & \\
\hline $\begin{array}{l}\text { Positive hyperabduction } \\
\text { maneuver }\end{array}$ & 30 & 34 & \\
\hline $\begin{array}{l}\text { Positive costoclavicular } \\
\text { maneuver }\end{array}$ & 29 & 35 & \\
\hline Scalene block & & & 0.54 \\
\hline Total no. performed & 21 & 27 & \\
\hline Positive & 15 & 17 & \\
\hline Negative & 6 & 10 & \\
\hline \multicolumn{4}{|l|}{ Scores before op } \\
\hline DASH (30 items) & $51.39 \pm 10.74$ & $53.38 \pm 9.63$ & 0.41 \\
\hline DASH subscale (6 items) & $18.52 \pm 2.28$ & $18.90 \pm 3.38$ & 0.87 \\
\hline Median FU in mos (range) & $36(12-67)$ & $32.5(12-65)$ & 0.43 \\
\hline
\end{tabular}

EAST = elevated arm stress test; FU = follow-up; ULTT = upper limb tension test.

Values expressed as the mean \pm standard deviation, median (range), or number.

tions, and the brachial plexus was surrounded with high signal intensity of fat as in healthy subjects. For all 33 patients in the sparing group, there were still signs of nerve impingement from the untreated subclavius on hyperabduction. However, the 3 patients in the sparing group who
TABLE 2. Functional outcomes of the subclavius sparing and dividing groups

\begin{tabular}{lccc}
\hline \multicolumn{1}{c}{ Assessment } & $\begin{array}{c}\text { Subclavius } \\
\text { Sparing } \\
\text { Group (33) }\end{array}$ & $\begin{array}{c}\text { Subclavius } \\
\text { Dividing } \\
\text { Group (38) }\end{array}$ & $\begin{array}{c}\mathrm{p} \\
\text { Value }\end{array}$ \\
\hline Quantitative & & & \\
\hline DASH (30 items) & $29.94 \pm 9.96$ & $27.65 \pm 12.69$ & 0.41 \\
\hline DASH subscale (6 items) & $11.94 \pm 2.87$ & $9.50 \pm 2.76$ & 0.0005 \\
\hline Qualitative & & & 0.73 \\
\hline Resolved & 2 & 4 & \\
\hline Markedly improved & 19 & 24 & \\
\hline Fair & 10 & 9 & \\
\hline Poor & 2 & 1 & \\
\hline
\end{tabular}

Boldface type indicates statistical significance.

previously had radiological compression in the neutral position no longer had such signs in the neutral position. Perhaps this occurred because the FRR left a groove in which the brachial plexus fell and lay freely when the arms were put alongside the body, and the brachial plexus was no longer in contact with the subclavius in the neutral position. Upon hyperabduction, however, the brachial plexus went up along with the arm and still became encroached upon by the subclavius above it.

\section{Influence of Treatment Approach on the DASH Subscale Score}

Univariate analysis demonstrated significant correlations between the postoperative subscale score and four different parameters: 1) the duration of the presence of symptoms prior to surgery $(r=0.30, p=0.01), 2)$ the type of treatment $(\mathrm{p}=0.0005), 3)$ the sex of the patients $(\mathrm{p}=$ $0.07)$, and 4$)$ the age of patients $(r=0.21, p=0.09$; Supplemental Table). Multivariate analysis revealed two independent factors influencing the DASH subscale scores: the type of treatment (standardized beta coefficients $=0.41$; $\mathrm{p}=0.000$ ) and the duration of the presence of symptoms prior to surgery (standardized beta coefficients $=0.30 ; p$ $=0.005$ ).

\section{Discussion}

In this study, we found that dividing rather than sparing the radiological nerve-compressing subclavius muscle in NTOS patients can lead to better functional recovery with respect to overhead activities as assessed by the DASH subscale. Postoperative radiological findings agreed with the different functional outcomes. When the arm was hyperabducted, patients in the sparing group still had signs of nerve impingement by the untreated subclavius, whereas the dividing group no longer had signs of nerve compression in the costoclavicular space given the resection of both the first rib and the subclavius. However, these two different surgical approaches did not significantly affect the surgical outcome as reflected by the DASH score and patient self-assessments.

After adjusting for covariates, the type of treatment (dividing vs sparing) remained an independent factor influ- 


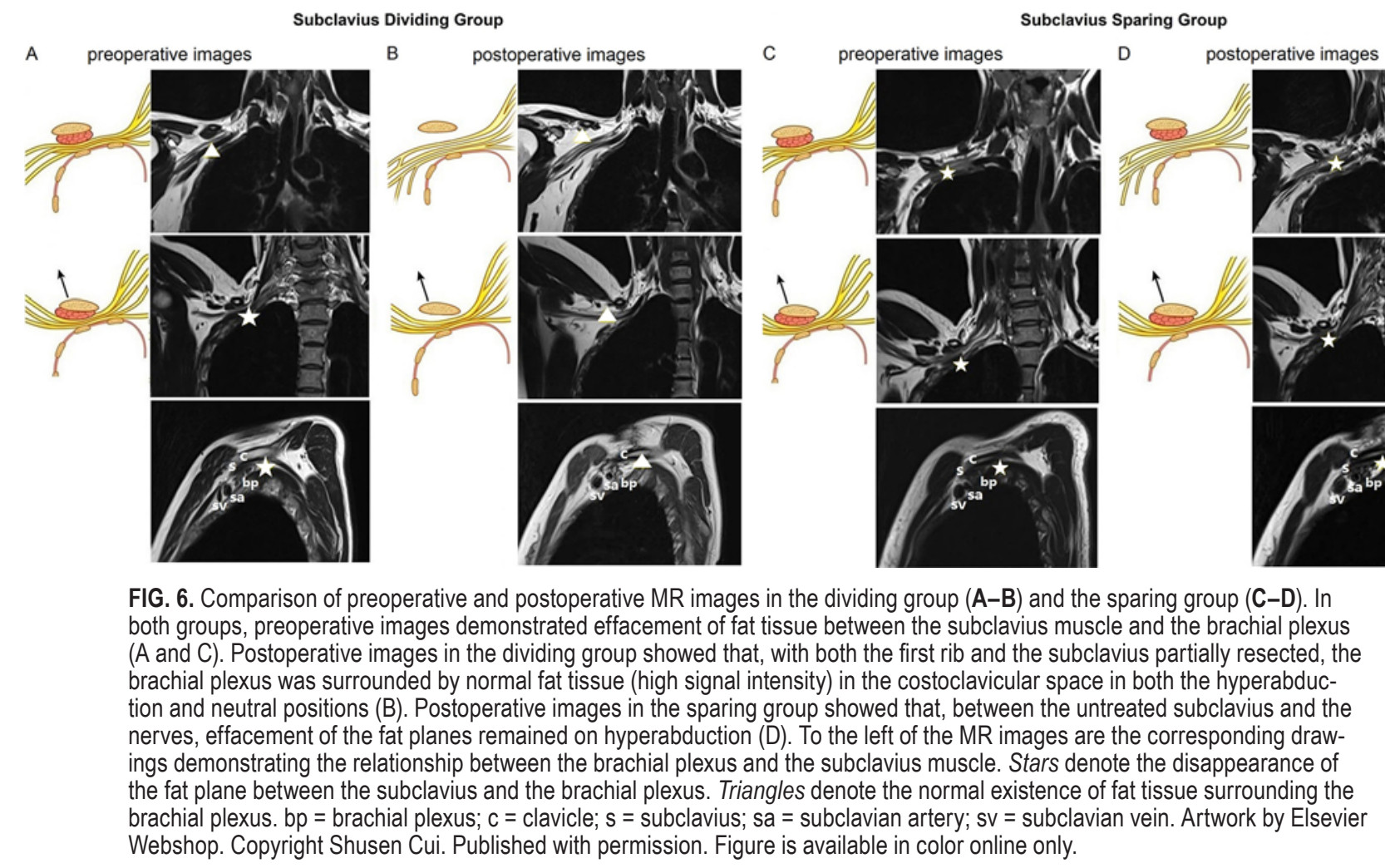

encing the DASH subscale scores. This suggested that the untreated radiological nerve-compressing subclavius may be associated with a lower degree of recovery in the ability to perform overhead activities. The subclavius muscle in this situation may participate in positional brachial plexus compression upon movements narrowing the costoclavicular space. However, to accurately predict whether a radiological nerve-compressing subclavius muscle is the true offending factor in any individual NTOS patient, further research is required to obtain more insight into the correlation between this imaging finding and NTOS symptomatology.

\section{Possible Pathophysiological Mechanisms of Brachial Plexus Impingement by the Subclavius}

The costoclavicular space is bounded superiorly by the clavicle and the subclavius muscle and inferiorly by the first rib. It is known that movements causing a scissor-like approximation of the first rib and the clavicle (e.g., arm hyperabduction, backward and downward bracing of the shoulder) can dynamically trap the brachial plexus in this tunnel. Most previous investigators have incriminated the first rib and performed FRR to decompress this space primarily.

Findings from more recent studies have suggested that subclavius muscles may also participate in nerve impingement in this tunnel via two possible mechanisms. The first is by occupying the space of the costoclavicular interval. The subclavius muscle arises by a short tendon from the costochondral junction of the first rib and runs laterally to the inferior middle third of the clavicle, with the brachial plexus passing beneath it. Its anatomical variations, such as anomalous coursing, fibrosis, or hypertrophy, could lead to a crowded costoclavicular space resulting in nerve compression. $6,7,14,16,24,26$

Second, the subclavius can narrow the costoclavicular interval by drawing the clavicle downward. Several groups have added subclavius blocks with botulinum as nonsurgical treatment, as it has been hypothesized that relaxing the muscle pulling down the clavicle while inducing its atrophy could decompress the costoclavicular space. ${ }^{18,33,34}$ Current treatments for NTOS include nonsurgical treatments such as PT and scalene muscle block with botulinum and thoracic outlet decompression surgeries. ${ }^{20} \mathrm{~A}$ few studies have reported improved outcomes after modified treatments involving the subclavius. ${ }^{2,18}$ One group from UCLA has reported an increased percentage of good outcomes (50\% or greater reduction in pain scores) after adding subclavius blocks for their patients with cervical brachial pain and limb paresthesia. ${ }^{18}$ These authors suggested that scalene blocking could allow the first rib, the "floor" of the costoclavicular space, to fall away from the brachial plexus, whereas blocking the subclavius allowed the clavicle, the "roof," to lift, thus providing additional decompression at the costoclavicular space. ${ }^{18}$ However, because of the concurrent addition of pectoral minor muscle blocks, uncertainty remains about the beneficial effect of subclavius blocks. In another study, Borrelly and Merle found that among 104 cases, 28 had hypertrophic subclavius muscles, which were subsequently resected through an additional deltopectoral groove incision resulting in satisfactory results for $95 \%$ of the patients, which had not been achieved with other approaches. ${ }^{2}$ However, the contribution of subclavius resection in that study was uncertain because of 
the concomitant slightly changed techniques pertaining to scalenectomy and FRR. ${ }^{2}$

To have a clear idea of whether treating the subclavius muscle can benefit surgical outcome, the two groups in our study received the same treatments in all respects except in terms of handling the subclavius muscle. Our comparative study demonstrated that though FRR could leave a groove in which the brachial plexus fell and lay freely while the arm was in the neutral position, upon arm hyperabduction, the plexus that went up with the arm still showed signs of impingement by the untreated subclavius. On the other hand, in patients in the dividing group, edges of the divided subclavius would retract, the muscle would atrophy, and the brachial plexus would become surrounded by fat in the costoclavicular space in both positions. Such findings indicated the association between elimination of the space-occupying effect of the subclavius and the better performance of movements that narrow the costoclavicular space, which corroborated the hypothesis raised in previous reports.

\section{Value of MRI in Evaluating the Role of the Subclavius in NTOS}

Some researchers consider MRI studies that demonstrate the proximity of the brachial plexus to adjacent structures with the effacement of fat planes as diagnostic of nerve compression. ${ }^{910,36}$ However, clinicians still hold different opinions on the use of MRI for etiological assessment of NTOS given the lack of information on the correlation between imaging findings and NTOS symptomatology. Even though we cannot reliably predict for any individual NTOS patient whether the nerve-compressing subclavius muscle is the cause of their NTOS symptomology, the results of our study, taken as a whole, suggest that such patients may benefit from subclavius division. In addition, MRI can identify a subclavius muscle without any contact with the brachial plexus, which does not need to be treated. One study blocked only the subclavius narrowing the costoclavicular space and encroaching upon the nerves, as demonstrated on MRI, thus saving the trouble of blocking the subclavius that was unlikely to be involved in NTOS. ${ }^{33}$

Regarding the imaging planes used for analysis, the coronal or sagittal plane has typically been used independently in previous studies. $1,8,27,32,35$ Coronal sequences provide better visualization of the course of the brachial plexus and the nerve impingement, ${ }^{27,35}$ but they can be subjective depending on the selected slice, resulting in a contradictory diagnosis if different slices are examined. ${ }^{32}$ Sagittal slices, on the other hand, are easier to standardize and less observer dependent. ${ }^{32}$ We contend that both coronal and sagittal views should be examined to evaluate the relationship between the subclavius muscle and the brachial plexus more accurately. In our experience, fat tissue between the subclavius and the brachial plexus sometimes disappeared on some sagittal slices, and coronal views showed that the subclavius touched the nerves at only a single point (Supplemental Fig. 2). However, such contact at a single point seemed more like "adjacency" rather than "impingement." Therefore, the effacement of fat tissue on at least three consecutive coronal sequences (a length of 9 $\mathrm{mm}$ according to section thickness) was required to minimize false-positives.

\section{Implications on Surgical Decision-Making for Thoracic Outlet Decompressions}

Supraclavicular and transaxillary decompressions are most widely used for NTOS, each having advantages and disadvantages. ${ }^{22}$ Some surgeons choose the approach based on what they are comfortable with and/or how they are trained, ${ }^{30}$ while others base their choice on the presence of bony anomalies, symptoms of upper or lower brachial plexus compression, or signs of neurogenic pectoralis minor syndrome. ${ }^{30}$

The implications of our results on the surgical decisionmaking process should be considered according to the decompression approach to be used and whether preoperative MRI is routinely performed. When the supraclavicular approach was applied together with routine MRI assessment, our findings suggested that dividing the subclavius with nerve-compressing signs could more adequately decompress the costoclavicular space. Dividing the subclavius through this modified supraclavicular approach is a rather safe procedure. No major complications occurred in patients in the dividing group with cautious blunt dissection and hemostasis.

However, regarding the supraclavicular approach used in the absence of preoperative MRI, this study has not provided sound evidence to support subclavius division, as the proportion of radiologically positive cases seemed to be small (less than 40\%) and the difference in the DASH score and self-assessments between the two groups was insignificant. This also indicated that a radiologically nerveimpinging subclavius was not as crucial as other wellknown factors that were much closer to the brachial plexus, such as scalene muscles and first ribs, and that it might only cause dynamic compression during overhead activities and would not severely affect most everyday activities.

\section{Postoperative Functional Assessments}

Both DASH and DASH subscale questionnaires were employed to assess postoperative functional outcomes. The DASH questionnaire is commonly used to measure physical function and symptoms in patients with musculoskeletal disorders of the upper limb including NTOS. Several studies have confirmed the multidimensionality of the DASH..$^{5,11,12,21}$ One study established through factor analysis the presence of three underlying subscales relating to manual functioning (items $1-5,7-11,16-18,20,21$ ), shoulder range of motion (items $6,12-15,19$ ), and symptoms and consequences (items 22-30). ${ }^{12}$

The shoulder range of motion subscale was used in our study because all its parameters involve overhead activities, which can significantly narrow the costoclavicular space resulting in proximity between the nerves and the subclavius muscle. Parameters assessed in the remaining DASH items are related to function and symptoms in the neutral position, and in this position, there is usually comfortable space between the subclavius and brachial plexus, as demonstrated in our imaging results. Therefore, we speculate that the DASH subscale can differentiate the functional difference better than the whole DASH survey. 
A minimal clinically important difference (MCID) exists for the overall DASH survey, ${ }^{15}$ but the MCID value for the subscale has not been studied. Therefore, it is uncertain whether the statistical significance shown by the subscale scores is clinically significant. Moreover, although the subscale is validated based on a scientific factor analysis of DASH, ${ }^{12}$ it has not been used in NTOS clinical research.

\section{Study Limitations}

First, this is a nonrandomized study with a small sample size. We considered the decision regarding the surgical approach was better made by the patients because dividing subclavius muscles via our modified approach was a preliminary exploration and its superiority in such patients had not been proven. Various factors might have prompted a patient to opt for a dividing or a sparing procedure, such as cosmetic considerations, severity of preoperative symptoms, and patient trust in our exploratory procedure. Therefore, intergroup homogeneity could not be ensured.

Second, a considerable proportion of the cases were traditionally called "disputed" NTOS. The lack of objective tests and unified diagnostic criteria for this subset of NTOS also poses a high risk of bias. ${ }^{29}$

Third, patients who could not endure MRI examination on hyperabduction were excluded because of incomplete imaging information. This could have caused a selection bias, as we may have missed out on patients with positive radiographic signs.

Fourth, functional assessment may have been influenced by a patient's occupation and avocational interests. For instance, athletes, who are susceptible to NTOS because of repetitive upper extremity use, usually have higher expectations for surgical outcomes, as they anticipate a return to their previous high performance level. ${ }^{4}$ However, a considerable proportion of the patients from our study were office workers and housewives who were not often involved with vigorous overhead use of their arms. Therefore, future studies can aim to identify whether certain subgroups are more likely to benefit from subclavius resection.

\section{Conclusions}

This study revealed that NTOS patients with a radiological nerve-compressing subclavius muscle may benefit from subclavius resection in terms of better recovery in the ability to perform overhead activities, indicating that such subclavius muscles may participate in positional nerve compression during movements narrowing the costoclavicular space. Therefore, dividing such subclavius muscles along with resecting the first rib could provide a more adequately decompressed costoclavicular space.

\section{Acknowledgments}

This study was supported by a grant from the Key Clinical Program of the Ministry of Health of China (No. [2010] 439; S.C.). Cost of postoperative MRI reexamination (around US\$110 at our institution) was covered by grant funds provided by the Ministry of Health of China.

\section{References}

1. Aralasmak A, Cevikol C, Karaali K, Senol U, Sharifov R,
Kilicarslan R, et al: MRI findings in thoracic outlet syndrome. Skeletal Radiol 41:1365-1374, 2012

2. Borrelly J, Merle M: [New surgical therapeutic approach for neurogenic thoracic outlet syndrome. "Saving the first rib". A homogeneous continuous series of 104 cases (July 2010-May 2013).] Chir Thorac Cardiovasc 18:143-148, 2014 (Fr)

3. Brantigan CO, Roos DB: Etiology of neurogenic thoracic outlet syndrome. Hand Clin 20:17-22, 2004

4. Chandra V, Little C, Lee JT: Thoracic outlet syndrome in high-performance athletes. J Vasc Surg 60:1012-1018, 2014

5. Cheng HM, Sampaio RF, Mancini MC, Fonseca ST, Cotta RM: Disabilities of the arm, shoulder and hand (DASH): factor analysis of the version adapted to Portuguese/Brazil. Disabil Rehabil 30:1901-1909, 2008

6. Cliffton EE: Neurovascular syndrome of the arm associated with hypertrophied subclavius muscle; report of a case, including operative treatment. Arch Surg 55:732-742, 1947

7. Cogar AC, Johnsen PH, Potter HG, Wolfe SW: Subclavius posticus: an anomalous muscle in association with suprascapular nerve compression in an athlete. Hand (N Y) 10:76-79, 2015

8. Demirbag D, Unlu E, Ozdemir F, Genchellac H, Temizoz O, Ozdemir H, et al: The relationship between magnetic resonance imaging findings and postural maneuver and physical examination tests in patients with thoracic outlet syndrome: results of a double-blind, controlled study. Arch Phys Med Rehabil 88:844-851, 2007

9. Demondion X, Bacqueville E, Paul C, Duquesnoy B, Hachulla E, Cotten A: Thoracic outlet: assessment with MR imaging in asymptomatic and symptomatic populations. Radiology 227:461-468, 2003

10. Demondion X, Boutry N, Drizenko A, Paul C, Francke JP, Cotten A: Thoracic outlet: anatomic correlation with MR imaging. AJR Am J Roentgenol 175:417-422, 2000

11. Fayad F, Lefevre-Colau MM, Macé Y, Fermanian J, MayouxBenhamou A, Roren A, et al: Validation of the French version of the disability of the arm, shoulder and hand questionnaire (F-DASH). Joint Bone Spine 75:195-200, 2008

12. Franchignoni F, Giordano A, Sartorio F, Vercelli S, Pascariello B, Ferriero G: Suggestions for refinement of the Disabilities of the Arm, Shoulder and Hand Outcome Measure (DASH): a factor analysis and Rasch validation study. Arch Phys Med Rehabil 91:1370-1377, 2010

13. Gambhir RP, Valenti D, Rashid H: Compliance with physical therapy is a key determinant of success of thoracic outlet surgical decompression. J Am Coll Surg 221:778, 2015

14. Georgiev GP, Jelev L: Bilateral fibrous replacement of subclavius muscle in relation to nerve and artery compression of the upper limb. IJAV 2:57-59, 2009

15. Gummesson C, Atroshi I, Ekdahl C: The disabilities of the arm, shoulder and hand (DASH) outcome questionnaire: longitudinal construct validity and measuring self-rated health change after surgery. BMC Musculoskelet Disord 4:11-16, 2003

16. Hasan SS, Romeo AA: Thoracic outlet syndrome secondary to an anomalous subclavius muscle. Orthopedics 24:793794,2001

17. Illig KA, Donahue D, Duncan A, Freischlag J, Gelabert H, Johansen K, et al: Reporting standards of the Society for Vascular Surgery for thoracic outlet syndrome. J Vasc Surg 64:e23-e35, 2016

18. Jordan SE, Ahn SS, Gelabert HA: Combining ultrasonography and electromyography for botulinum chemodenervation treatment of thoracic outlet syndrome: comparison with fluoroscopy and electromyography guidance. Pain Physician 10:541-546, 2007

19. Kim DH, Hudson AR, Kline DG: Thoracic outlet syndrome, in Kim DH (ed): Atlas of Peripheral Nerve Surgery, ed 2. Philadelphia: Elsevier/Saunders, 2013, pp 91-94 
20. Kuhn JE, Lebus V GF, Bible JE: Thoracic outlet syndrome. J Am Acad Orthop Surg 23:222-232, 2015

21. Lee EW, Chung MM, Li AP, Lo SK: Construct validity of the Chinese version of the disabilities of the arm, shoulder and hand questionnaire (DASH-HKPWH). J Hand Surg Br 30:29-34, 2005

22. Lee JT: Thoracic outlet syndrome: neurogenic, in Cronenwett JL (ed): Rutherford's Vascular Surgery, ed 8. Philadelphia: Elsevier, 2014, pp 1951-1968

23. Lum YW, Freischlag JA: Thoracic outlet syndrome: general considerations, in Cronenwett JL (ed): Rutherford's Vascular Surgery, ed 8. Philadelphia: Elsevier, 2014, pp 1936-1950

24. Martin RM, Vyas NM, Sedlmayr JC, Wisco JJ: Bilateral variation of subclavius muscle resembling subclavius posticus. Surg Radiol Anat 30:171-174, 2008

25. Merle M, Borrelly J, Villani F, Parra L: [Surgical treatment of neurogenic thoracic outlet syndrome.] E-Mémoires de l'Académie Nationale de Chirurgie 10:84-94, 2011 (Fr)

26. Muellner J, Kaelin-Lang A, Pfeiffer O, El-Koussy MM: Neurogenic thoracic outlet syndrome due to subclavius posticus muscle with dynamic brachial plexus compression: a case report. BMC Res Notes 8:351-353, 2015

27. Panegyres PK, Moore N, Gibson R, Rushworth G, Donaghy M: Thoracic outlet syndromes and magnetic resonance imaging. Brain 116:823-841, 1993

28. Plewa MC, Delinger M: The false-positive rate of thoracic outlet syndrome shoulder maneuvers in healthy subjects. Acad Emerg Med 5:337-342, 1998

29. Povlsen B, Hansson T, Povlsen SD: Treatment for thoracic outlet syndrome. Cochrane Database Syst Rev (11):CD007218, 2014

30. Powell A, Illig KA: Neurogenic thoracic outlet syndrome, in Tubbs RS (ed): Nerves and Nerve Injuries. Philadelphia: Elsevier, 2015, Vol 2, pp 709-723

31. Roos DB: Experience with first rib resection for thoracic outlet syndrome. Ann Surg 173:429-442, 1971

32. Smedby O, Rostad H, Klaastad O, Lilleås F, Tillung T, Fosse E: Functional imaging of the thoracic outlet syndrome in an open MR scanner. Eur Radiol 10:597-600, 2000

33. Torriani M, Gupta R, Donahue DM: Botulinum toxin injection in neurogenic thoracic outlet syndrome: results and expe- rience using a ultrasound-guided approach. Skeletal Radiol 39:973-980, 2010

34. Tran AT, Moguel-Cobos G, Koopot R, Lieberman A: Botulinum neurotoxin for neurogenic thoracic outlet syndrome. Toxicon 93:S60-S61, 2015

35. Van Es HW, Witkamp TD, Ramos LMP, Feldberg MAM, Nowicki BH, Haughton VM, et al: MR imaging of the brachial plexus using a T1-weighted three-dimensional volume acquisition. Int J Neuroradiol 2:264-273, 1996

36. Werden S: Radiographic imaging in diagnosis and assessment of NTOS, in Illig KA (ed): Thoracic Outlet Syndrome. London: Springer-Verlag, 2013, pp 111-125

\section{Disclosures}

The authors report no conflict of interest concerning the materials or methods used in this study or the findings specified in this paper.

\section{Author Contributions}

Conception and design: Liu. Acquisition of data: Zhang, Wang, Wu. Analysis and interpretation of data: Zhang, Wang, Wu, Yu. Drafting the article: Liu. Critically revising the article: Cui, Liu. Reviewed submitted version of manuscript: Zhang. Approved the final version of the manuscript on behalf of all authors: Cui. Statistical analysis: Wang, Wu, Yu. Administrative/technical/material support: Yu. Study supervision: Liu, Zhang.

\section{Supplemental Information \\ Online-Only Content}

Supplemental material is available with the online version of the article.

Supplemental Material. https://thejns.org/doi/suppl/10.3171/ 2018.5.JNS18429.

\section{Correspondence}

Shusen Cui: China-Japan Union Hospital of Jilin University, Changchun, China. cuiss@jlu.edu.cn. 